\title{
Effect of Droplet Distortion on Surface Tension in Electromagnetic Levitation Method
}

\author{
K. Nogi, T. Nakano, T. Matsumoto, and H. Fujii \\ Joining and Welding Research Institute, Osaka University, 11-1 Mihogaoka, Ibaraki Osaka, Japan
}

\begin{abstract}
The surface tension of molten Si was measured using the electromagnetic levitation method under a microgravity condition. The experiments were carried out over a wide temperature range between $1460 \mathrm{~K}$ (below the melting point) and $1880 \mathrm{~K}$. The atmosphere was $\mathrm{Ar}+3 \% \mathrm{H}_{2}$ purified by Platinum asbestos and magnesium perchlorate. The oxygen partial pressure is estimated to be at least than $1.1 \times 10^{-14} \mathrm{~Pa}$. The shape of the droplet is controlled by changing the output ratio for two different coils. The equilibrium shape of the oscillating droplet was precisely determined by calibrating the apparent change in the droplet shape. The effect of the droplet distortion on the shift in the oscillation frequencies and the calculated surface tension value was also investigated. When the equilibrium shape of the levitating droplet is spherical, the surface tension of molten Si was obtained with very small scatter. The value is expressed as follows : $\gamma=-0.074(T-1687)+735 \gamma$ : Surface tension $(\mathrm{mN} / \mathrm{m}), T$ : Temperature(K).
\end{abstract}

KEY WORDS : microgravity; electromagnetic levitation; surface tension; droplet shape; molten silicon

\section{Introduction}

The electromagnetic levitation method is very useful for measuring the surface tension of molten materials. Because molten materials are generally highly reactive at high temperatures, the surface tension of pure molten materials cannot be easily measured with high accuracy using conventional methods. In the electromagnetic levitation method, however, the sample does not come into contact with any crucible or substrate, which are usually the major source of contamination ${ }^{1,2)}$. This method also allows the surface tension to be measured in the undercooled state due to the absence of nucleation sites. However, under terrestrial conditions, the accuracy of the surface tension obtained by this method is not very high because a droplet is distorted from a sphere due to gravity and the balanced magnetic force. To redress this problem, a microgravity condition is very effective, where the shape of the levitated droplet is close to a sphere and can be more easily controlled. It is thought that the equilibrium shape of the droplet should significantly affect the oscillation condition. However, the relationship has never been discussed in detail. In this study, the shape of the droplet is controlled by changing the output ratio for two different coils and the effect of the distribution on the oscillation frequency is investigated.

\section{Measurement Principle}

A levitated sample oscillates around the equilibrium shape. When the equilibrium shape is spherical, the surface tension can be calculated using the following equation ${ }^{3)}$ :

$$
\omega_{l}^{2}=\frac{4}{3} \pi l(l-1)(l+2) \frac{\gamma}{M}
$$

where $\omega$ is the angular frequency of the surface oscillation, and $l$ is the label for the oscillation modes. $M$ is the droplet mass, and $\gamma$ is the surface tension. The frequency of $l=2$ is called the Rayleigh frequency and can be written as follows:

$$
v_{R}^{2}=\frac{8}{3 \pi} \frac{\gamma}{M}
$$

where $\omega=2 \pi v_{R}$. In practice, however, the equilibrium shape is not spherical and, therefore, a frequency spectrum with five peaks is obtained. In this case, Cummings \& Blackburn's correction formula could be used to calculate the Rayleigh frequency ${ }^{4}$ ):

$$
\begin{gathered}
\omega_{R}^{2}=\frac{1}{5}\left(\omega_{l-2, m-0}^{2}+2 \omega_{l-2, m-1}^{2}+2 \omega_{l-2, m-2}^{2}\right) \\
-\omega_{t r}^{2}\left\{1.90+1.20\left(\frac{z_{0}}{a}\right)^{2}\right\} \\
z_{0}=\frac{g}{2 \omega^{2}}
\end{gathered}
$$

where $\omega_{t r}$ is the mean translation frequency of the drop's center of mass, $g$ is the gravitational acceleration, and $a$ is the radius of the droplet, while $l$ and $m$ are the labels for the oscillation modes.

\section{Experimental Procedure}

Figure 1 shows a schematic diagram of the experimental apparatus. This system has a specially designed coil system. It consists of three types of coils. The positioning coil is used to levitate the sample, the heating coil is used to heat the sample and the detecting coil is used to detect the droplet position.

The silicon samples used were of $9 \mathrm{~N}$ purity and weighed about $1.2 \mathrm{~g}$ or $0.7 \mathrm{~g}$. The sample was preheated with an infrared ray heater, because $\mathrm{Si}$ has a high electric resistance 


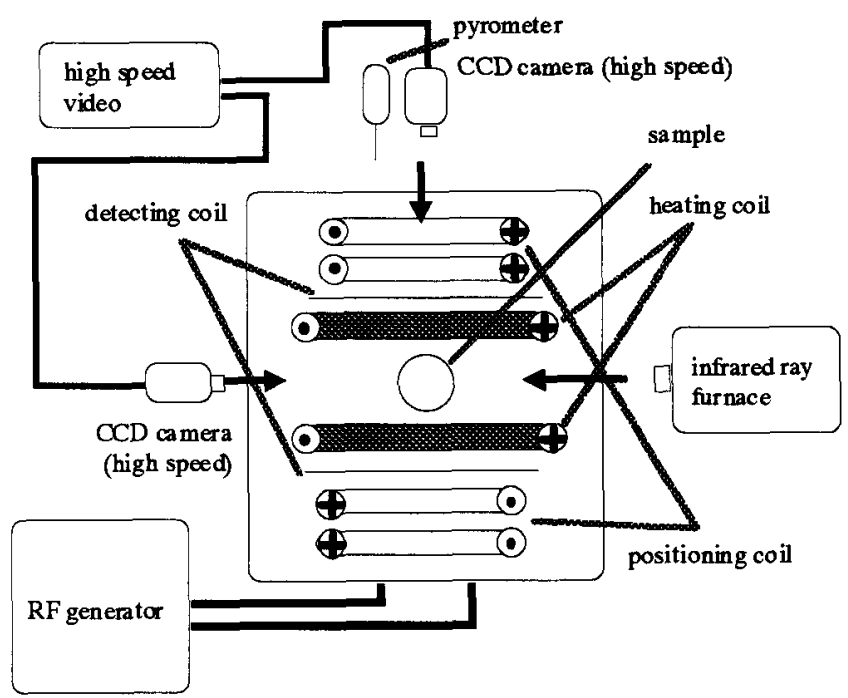

Fig. 1 Schematic illustration of experimental apparatus.

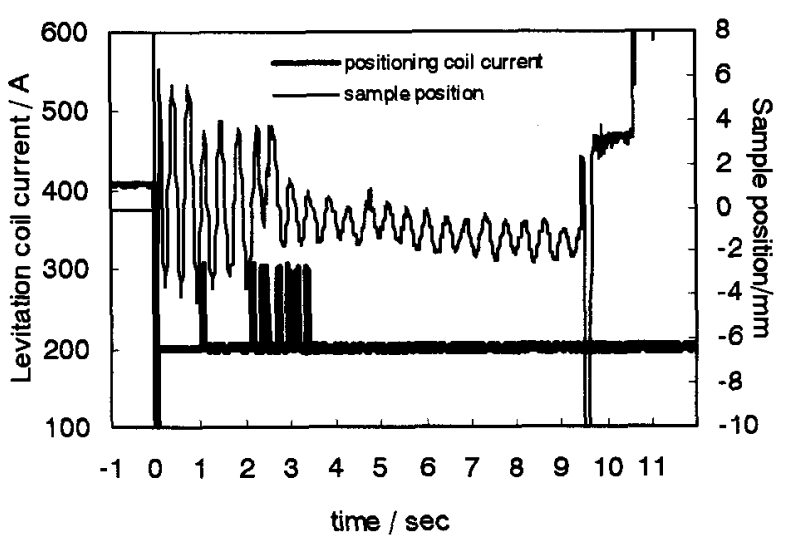

Fig. 2 Feedback control of sample oscillation in early stage of falling.

at room temperature and an eddy current cannot flow in the sample. It was then electromagnetically heated and levitated without contact with any crucible. The temperature of the levitated sample was measured with a two-color pyrometer. To obtain a microgravity condition the entire levitation system was dropped inside a free fall system. The drop shaft facility at the Japan Microgravity Center (JAMIC) was used in this study. The level of microgravity is about $10^{-5} \mathrm{G}$ with about 10 second duration. When the gravity changes from $1 \mathrm{G}$ to $10^{-5} \mathrm{G}$, the positioning coil current is adjusted to maintain the droplet in the middle of the coil using the detecting coil data. An example of the changes in the positioning coil current and the sample translation is shown in Fig.2. The translation of the droplet was damped in the early stage of the fall. During the fall, the surface oscillation of the droplet was recorded by high-speed video cameras (200frame/sec) in both the horizontal and vertical directions. The frequency spectra are obtained from the recorded data by Fourier transformation. The surface tension was calculated by equation(2). When the frequency peaks are split, equation(3) was used. The flowing atmosphere was $\mathrm{Ar}+3 \% \mathrm{H}_{2}$ purified using $\mathrm{Pt}$ asbestos and magnesium perchlorate. It was confirmed by an analysis of the output gas that silicon is deoxidized in the purified gas at the melting point. Therefore, the oxygen partial pressure is at least less than $1.1 \times 10^{-14} \mathrm{~Pa}$.

A two-color pyrometer was used to measure the temperature of the droplet. When a pyrometer is used, the emissivity has to be determined. This value is usually determined at the melting point by adjusting the indicating temperature to the melting point. In this study, the degree of the error was measured when the emissivity determined at the melting point was assumed to be constant. The temperature of the molten silicon in an $\mathrm{Al}_{2} \mathrm{O}_{3}$ crucible was
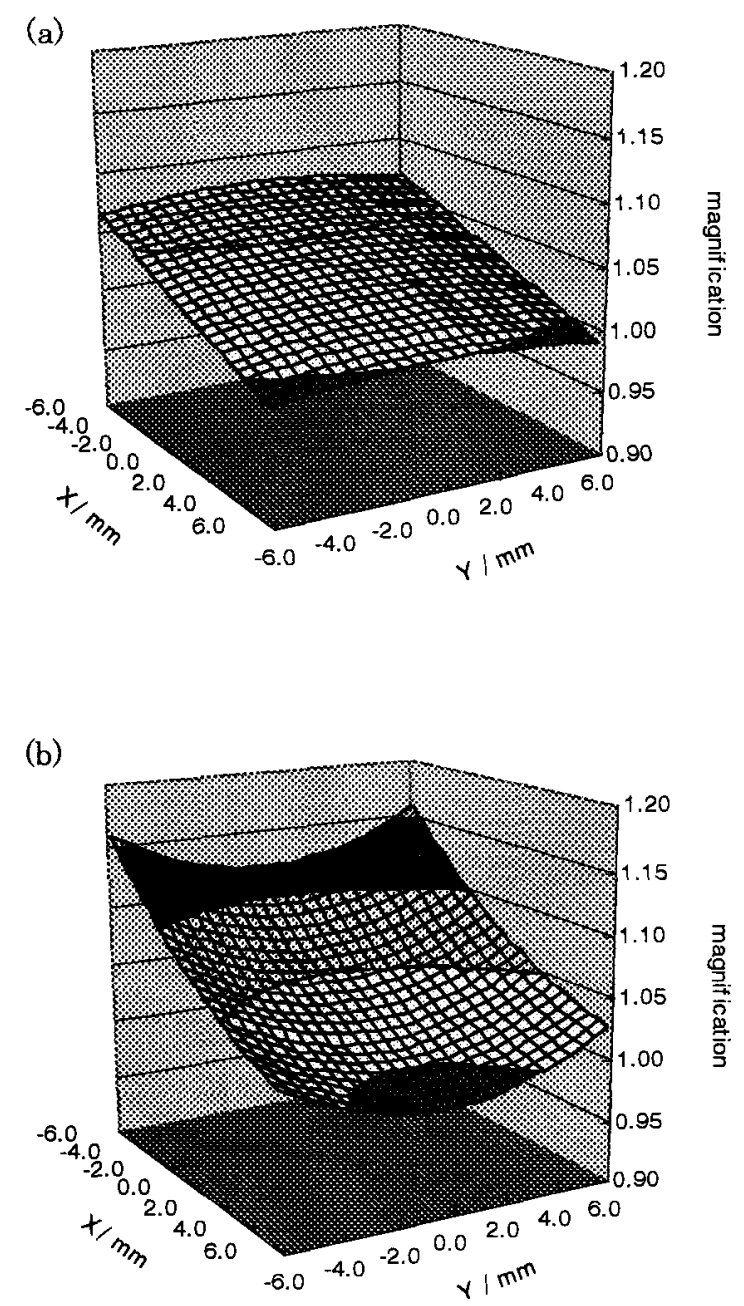

Fig . 3 Apparent diameter of droplet observed in horizontal direction.

(a) vertical diameter

(b) horizontal diameter

Scale sphere was observed from minus $X$ direction. $X=0, Y=0$ point is focusing position. 
measured with both the pyrometer and a thermocouple and then compared ${ }^{5}$.

When the droplet is not located at the focus position of the cameras, the apparent droplet size changes. In addition, when the droplet is observed in the horizontal direction, the droplet is recorded through a $\mathrm{SiO}_{2}$ tube, which has a curvature. In this case, the apparent shape as well as the size changes. Accordingly, the shape change with the droplet position was measured. Figs.3 (a) and (b) shows the apparent length of the horizontal diameter and vertical diameter of the droplet, respectively. The results were measured with a $10 \mathrm{~mm}$ solid silicon sphere at about $1680 \mathrm{~K}$ (about $10 \mathrm{~K}$ below the melting point). The calibration was made for each recorded frame using these data. In this case, the coordinate of the droplet position which is necessary for the calibration of the top view was determined with the side view data, and the coordinate for the side view was determined with the top view.
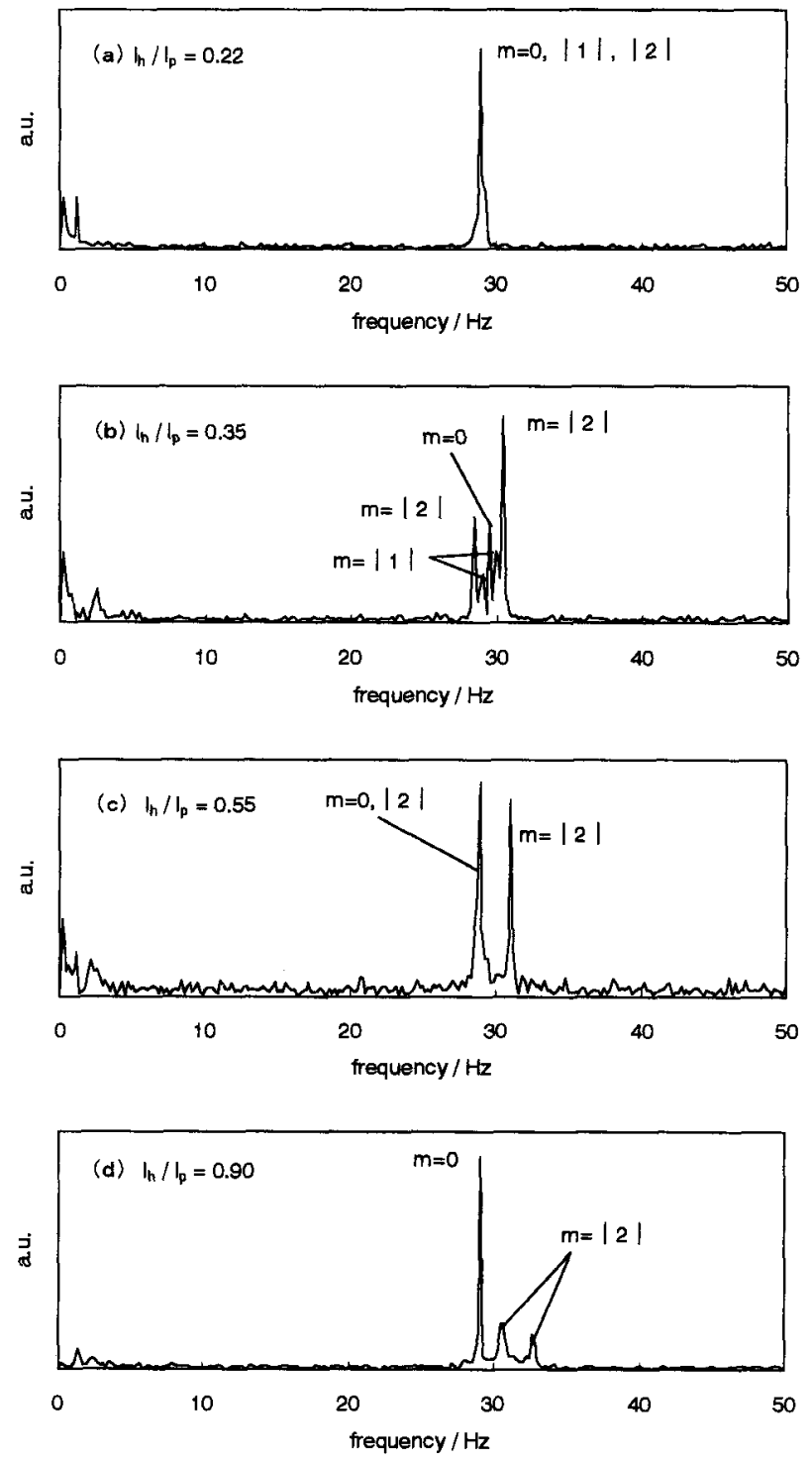

Fig. 4 Change in the frequency spectrum measured at various current ratio

\section{Results and Discussion}

The shape of the droplet is deformed to be oblate by the positioning coil, but prolate by the heating coil, and can be controlled by changing the strength of the magnetic field formed by the two coils. In order to investigate the effect of the droplet distortion on the surface oscillation, the surface oscillation was analyzed with changing the coil current ratio of the heating coil current $\left(I_{h}\right) /$ the positioning coil current $\left(I_{\mathrm{p}}\right)$ from 0 to 0.9 . Figure 4 shows the change in the frequency spectrum obtained from the top view. The current ratio, $I_{\mathrm{h}} / I_{\mathrm{p}}$, in each case is (a) 0.22 , (b) 0.35 , (c) 0.55 and (d) 0.9 . When $I_{\mathrm{h}} / I_{\mathrm{p}}$ is 0.22 , the equilibrium shape is closest to a sphere and the frequencies of the five oscillations are the same, and consequently, they look only one peak in the spectrum. (b) When $I_{\mathrm{h}} / I_{\mathrm{p}}$ is 0.35 , the shape is slightly distorted from a sphere, and all the five fundamental oscillations are detected. (c) When $I_{\mathrm{h}} / I_{\mathrm{p}}$ is 0.55 , the shape is more distorted and three strong peaks of the $l=2, m=0$ and $l=2, m= \pm 2$ modes are excited. The peak of $m=0$ shifts to a lower frequency and the peaks of $m= \pm 2$ shift to higher frequencies. (d) When $I_{\mathrm{h}} / I_{\mathrm{p}}$ is 0.9 , the shape is significantly distorted and a strong peak of the $l=2, m=0$ mode and two peaks of the $l=2, m= \pm 2$ mode are excited. The $m= \pm 1$ peaks are not usually observed for the top view. As can be seen in a series of these figures, the peak distribution is clearly dependent on the droplet shape. As the shape is distorted from a sphere, the surface oscillation frequency splits first and then particular modes are excited. When the droplet is distorted in the vertical direction as in this study, the $m=0$ mode shifts to a lower frequency, and the $m= \pm 2$ modes shift to a higher frequency. When the degree of the shape distortion is expressed by the ratio of the vertical diameter, $V$, / the horizontal diameter, $H$, of the droplet, the degrees, $V / H$ are $1.015,1.045$ and 1.14 for $I_{\mathrm{h}} / I_{\mathrm{p}}$ of $0.35,0.55$, and 0.9 , respectively.

Figure 5 shows the surface tension calculated using the data when the droplet is almost spherical. The thin straight lines are previously reported values ${ }^{2,6-14)}$. The

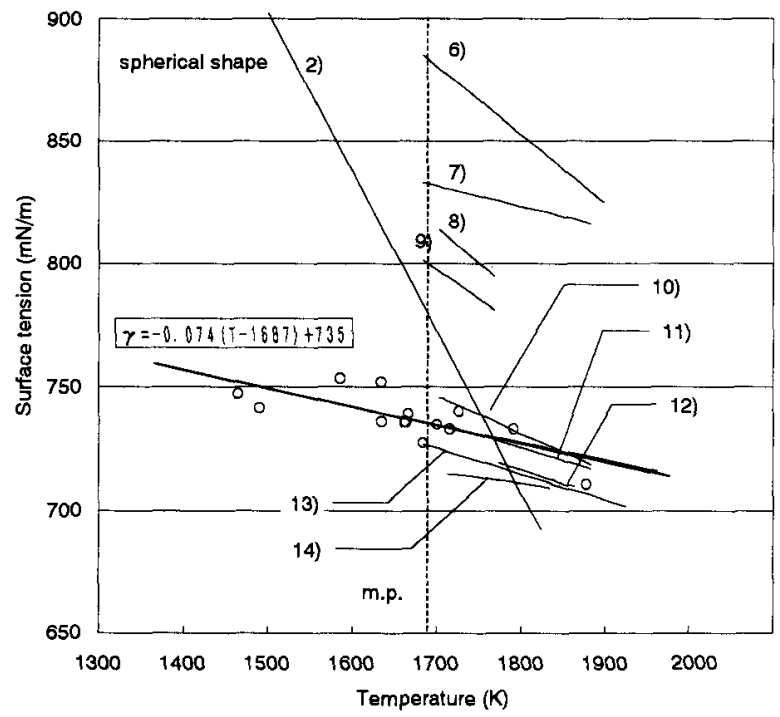

Fig 5 Surface tension of molten Si when the droplet is almost spherical 


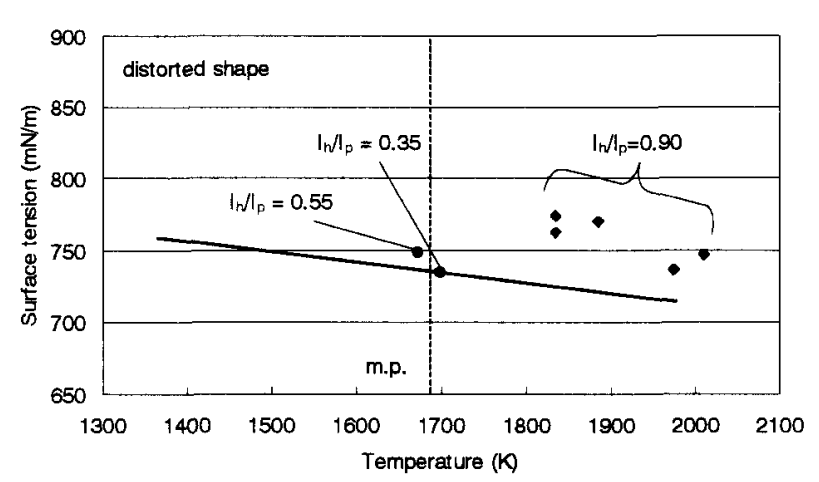

Fig.6 Surface tension of molten Si when the droplet is distorted from sphere

surface tension was measured over a wide temperature range between $1460 \mathrm{~K}$ (below the melting point) and $1880 \mathrm{~K}$. The temperature dependence is expressed by the following equation :

$$
\gamma=-0.074(T-1687)+735
$$

where $T$ is the temperature in $\mathrm{K}$. The relationship can be linearly approximated and the surface tension decreases with increasing temperature. Note that the scatter of the obtained surface tension values are much smaller than those by conventional methods. This is because the size determination is unnecessary and only the frequency and the droplet mass are used for the calculation.

As shown in Figure 5, there are large differences among the reported values. The value obtained in the present study is close to many previously reported values $^{10-14)}$, but is obviously smaller than those obtained by Hardy ${ }^{6)}$ and some others ${ }^{7,8,9}$. A possible cause for the difference is the difference in the oxygen contents at the sample surface. The surface tension decreases with increasing the oxygen partial pressure ${ }^{1,15)}$. However the difference between the present value and the value obtained by Przyborowski et $\mathrm{al}^{2)}$ cannot be explained by this reason. Although they used the electromagnetic levitation method (in a terrestrial condition) and the oxygen partial pressure in their study was higher than that in the present study, the surface tension value is larger than that in the present study.

In order to investigate the effect of the distortion of the droplet on the surface tension, the surface tension was calculated when the current ratio, $I_{\mathrm{b}} / I_{\mathrm{p}}$, was $0.35,0.55$, and 0.9. In this case, Cummings \& Blackburn's correction formula was used to calculate the surface tension. The results are shown in Fig. 6. As the current ratio is larger, namely, as the shape of the droplet becomes longer in the vertical direction, the surface tension value calculated using Cummings and Blackburn's equation tends to be larger. When the current ratio is 0.9 , the obtained values were approximately $7 \%$ larger. Thus, when the shape is too distorted, Cummings \& Blackburn's correction formula cannot be applied. In the terrestrial environment, the distortion of the droplet is generally larger than that in this study.

\section{Conclusions}

(1) The surface tension of liquid silicon was measured by controlling the droplet shape close to a sphere under microgravity. The atmosphere was $\mathrm{Ar}+3 \% \mathrm{H}_{2}$ purified by Platinum asbestos and magnesium perchlorate. The oxygen partial pressure is estimated to be at least less than $1.1 \times 10^{-14} \mathrm{~Pa}$. The values obtained over a wide temperature range are expressed by the following equation:

$$
\begin{gathered}
\gamma=-0.074(T-1687)+735 \\
\gamma: \text { surface tension }(\mathrm{mN} / \mathrm{m}), \quad T: \text { temperature }(\mathrm{K})
\end{gathered}
$$

(2) When the distortion of the droplet is small, the surface tension of the liquid can be calculated using Cummings \& Blackburn's equation. However, when the distortion is larger, the error in the calculated value is not negligible.

\section{Acknowledgements}

This work is the result of "Technology for Production of High Quality Crystal" which is supported by the New Energy and Industry Technology Development Organization (NEDO) through the Japan Space Utilization Promotion Center (JSUP) in the program of the Ministry of International Trade and Industry (MITI), and also of a Grant-in-aid for Scientific Research(A) and Encouragement of Young Scientist of the Ministry of Education, Science, Sports, and Culture.

\section{REFERRENCES}

1) B. J. Keene : Surface Interface Anal, $\mathbf{3 6 7 - 3 8 3 ~ ( 1 9 8 7 ) , ~} 10$.

2) M. Przyborowski, T. Hibiya, M. Eguchi and I. Egry : $J$. Crystal Growth, 60-65 (1995), 151.

3) Lord Rayleigh : Proc. Roy. Soc. London, $71-97$ (1879), 29.

4) D. Cummings and D. Blackburn : J. Fluid Mech., 395-416 (1991), 224.

5) H. Fujii, T. Matsumoto, N. Hata, T. Nakano, M. Kohno and K. Nogi, in press Metall. Mater. Trans. A.

6) S. C. Hardy : J. Crystal Growth, 456-460 (1984), 69.

7) В.Н.ВОБКОВСКИИ, ВИ.КОСТКОВ, В.ЛЕВИН А.С.ТАРАБАНОВ : Конструкбионнын, метерналына основе графита $M$, Металтургия 138-143 (1970), 5

8) S. I. Chung, K. Izunome, A. Yokotani and S.Kimura : Jpn. J. Appl. Phys., 631-634 (1995), 34, 5 b.

9) N. Kawasaki, K. Watanabe and Y. Nagasaka : High Temperatures - High Pressures, 91-96 (1998), 30.

10) Yu. V. Naidich, V. M. Perevertailio and L. P. Obshchak : Poroshkovaya Metallurguya, 73-75 (1975), 49, 5.

11) E. S. Levin, P. V. Gel'd and B. A. Baum : Russ. J. Phys. Chem., 1455-1459 (1966), 40, 11.

12) P. H. Keck and W. V. Horn : Phys. Rev., 512-513 (1953), 91, 3.

13) S. V. Lukin, V. I. Zhuchkov, N. A. Vatlin, Yu. S. Kozlov : J. Less-Common Metals, 407-413 (1979), 67.

14) S. I. Popel', L. M. Shergen, B. V. Tsarevskii : Russ. J. Phys. Chem., 144-145 (1970), 44, 1.

15) K. Mukai, Z. Yuan, K. Nogi and T. Hibiya : Proc of The Twentieth Japan Symposium on Thermophysical Properties 1999, Tokyo, 264-267 (1999) 